This item was submitted to Loughborough's Research Repository by the author.

Items in Figshare are protected by copyright, with all rights reserved, unless otherwise indicated.

\title{
Stochastic MPC for additive and multiplicative uncertainty using sample approximations
}

PLEASE CITE THE PUBLISHED VERSION

https://doi.org/10.1109/tac.2018.2887054

\section{PUBLISHER}

Institute of Electrical and Electronics Engineers (IEEE)

VERSION

AM (Accepted Manuscript)

\section{PUBLISHER STATEMENT}

Personal use of this material is permitted. Permission from IEEE must be obtained for all other uses, in any current or future media, including reprinting/republishing this material for advertising or promotional purposes, creating new collective works, for resale or redistribution to servers or lists, or reuse of any copyrighted component of this work in other works.

\section{LICENCE}

\section{All Rights Reserved}

\section{REPOSITORY RECORD}

Fleming, James, and Mark Cannon. 2018. "Stochastic MPC for Additive and Multiplicative Uncertainty Using Sample Approximations". Loughborough University. https://hdl.handle.net/2134/12623831.v1. 


\title{
Stochastic MPC for additive and multiplicative uncertainty using sample approximations
}

\author{
James Fleming Mark Cannon
}

\begin{abstract}
We introduce an approach for Model Predictive Control (MPC) of systems with additive and multiplicative stochastic uncertainty subject to chance constraints. Predicted states are bounded within a tube and the chance constraint is considered in a 'one step ahead' manner, with robust constraints applied over the remainder of the horizon. The online optimization is formulated as a chance-constrained program which is solved approximately using sampling. We prove that if the optimization is initially feasible, it remains feasible and the closed-loop system is stable. Applying the chance-constraint only one step ahead allows us to state a confidence bound for satisfaction of the chance constraint in closed-loop. Finally, we demonstrate by example that the resulting controller is only mildly more conservative than scenario MPC approaches that have no feasibility guarantee.
\end{abstract}

\section{INTRODUCTION}

Robust Model Predictive Control (MPC) is a method of controlling uncertain systems in which predictions of states are used to minimize a cost function online. Unlike linear feedback controllers, MPC allows inequality constraints to be handled systematically and, if suitable terminal constraints are included in the optimization, it can ensure that those constraints are satisfied for all uncertainty realizations. In general, propagating the effects of parametric or multiplicative uncertainty over a prediction horizon of length $N$ involves computation that grows exponentially with $N$. This difficulty can be avoided through the introduction of a prediction tube [1], [2]. Recent papers have provided convenient parameterizations of such tubes for systems with parametric uncertainty [3], [4].

In the presence of stochastic uncertainty, it is often desirable to limit the probability of constraint violations. In this case a robust approach, which enforces constraints for all uncertainty realizations, may be conservative. Instead, knowledge of the probability distribution of uncertainty should be used to relax constraints. For a linear system with additive uncertainty, constraint tightening/relaxing parameters may be calculated to handle chance constraints [5], [6] and the resulting controller guarantees constraint satisfaction and stability in closed-loop. No similar result exists for stochastic uncertainty in system parameters, and the aim here is to introduce a suitable framework by using conditions for a polyhedron to be contained in a set defined by chance constraints. This results in a receding horizon control problem whose solution can be approximated using a sample-based method, while providing closed-loop feasibility, stability and constraint satisfaction guarantees.

Corresponding author: j.m.fleming@soton.ac.uk. James Fleming is with the School of Engineering, University of Southampton, SO17 1BJ, UK. Mark Cannon is with the Department of Engineering Science, University of Oxford, OX1 3PJ, UK
In [7] and [8] a scenario approach is used for predictive control, where the probability that constraints are satisfied over some finite horizon is bounded. These scenario methods are very general, but they do not guarantee that the optimization problem remains feasible at subsequent times. In contrast, we consider 'one step ahead' chance constraints (as in [5] and [6]) and use a tube to bound the predicted state, which guarantees recursive feasibility if the uncertainty has compact support. This extends the work of [3] by introducing additive uncertainty, less conservative terminal conditions, and a closedloop confidence bound on constraint violation, and provides a stochastic counterpart to the robust controller of [4].

a) Notation: We use $\mathbb{E}_{t}[\cdot]$ to denote conditional expectation given state $x(t)$ while $\operatorname{Pr}_{t}[\cdot]$ denotes conditional probability given $x(t)$. The convex hull of a set of points is written as Co $\{\cdot\}$. To refer to the $i$ th row of a matrix $H$, we write $(H)_{i}$. For convenience when developing the controller, we consider the time $t=0$ and denote predictions of the input and state $u(t), x(t)$ at a future time $t=k$ as $u_{k}, x_{k}$.

\section{Problem Statement}

We consider the control of a discrete-time system,

$$
x(t+1)=A(t) x(t)+B(t) u(t)+w(t) .
$$

where the state $x(t)$ is available at each $t$ and $u(t)$ is a control input. The values $A(t), B(t), w(t)$ depend on a vector-valued stochastic process $q(t)$ with elements $q_{i}(t) \in \mathbb{R}, i=1 \ldots \mu$,

$$
(A(t), B(t), w(t))=\sum_{i=1}^{\mu}\left(A^{\{i\}}, B^{\{i\}}, w^{\{i\}}\right) q_{i}(t)
$$

for some known $A^{\{i\}}, B^{\{i\}}, w^{\{i\}}$, with $i=1, \ldots, \mu$. To simplify notation we write $A, B, w, q$ in place of $A(t), B(t)$, $w(t), q(t)$ wherever the time-dependence is clear. We briefly note that (II.2) allows $A, B$ and $w$ to be independent, as $q$ may be partitioned as $q=\left[\begin{array}{lll}q_{A} & q_{B} & q_{w}\end{array}\right]^{T}$ and, for example, $B^{\{i\}}=w^{\{i\}}=0$ for $i$ corresponding to elements of $q_{A}$.

Assumption 1. For any $t_{1} \neq t_{2}, q\left(t_{1}\right)$ and $q\left(t_{2}\right)$ are independent and identically distributed (i.i.d.).

Assumption 2. The probability distribution of $q$ is compactly supported with $q \in \operatorname{Co}\left\{q^{(1)}, \ldots, q^{(\nu)}\right\}$ almost surely.

Remark 1. Assumption 1 limits the approach to systems in which the uncertainty affecting $A(t)$ and $B(t)$ is i.i.d., in common with existing methods [3], [8], [9]. Temporal correlations in $w(t)$ can be handled by augmenting the system with a linear filter generating non-i.i.d. noise. Assumption 2 
is required for recursive feasibility, and is used to bound the uncertainty robustly after the first prediction time.

The control objective is to minimize the expected value of

$$
\sum_{k=t}^{\infty} \mathbb{E}_{t}[L(k)]=\sum_{k=t}^{\infty} \mathbb{E}_{t}\left[x(k)^{T} Q x(k)+u(k)^{T} R u(k)\right],
$$

where $Q$ and $R$ are positive definite matrices, while, for some probability level $p \in(0,1]$, satisfying constraints:

$$
\begin{gathered}
F_{h} x(t)+G_{h} u(t) \leq \underline{1} \\
\operatorname{Pr}_{t}\left[F_{p} x(t+1) \leq \underline{1}\right] \geq p .
\end{gathered}
$$

Constraint (II.5) limits the probability of a constraint violation at the next sample time using the most recent information available, which is useful in applications such as wind turbine control [9]. At each time $t$ the controller minimizes (II.3), which is the expected future performance given present information, subject to (II.4) and (II.5). We write $A^{(j)}, B^{(j)}$ and $w^{(j)}$ for values of $A, B, w$ in (II.2) corresponding to $q=q^{(j)}$.

\section{INPUT, STATE AND TUBE PARAMETRIZATION}

We parametrize the predicted control input as

$$
u_{k}=K x_{k}+c_{k}
$$

where $K$ is chosen so that the dynamics $\xi_{k+1}=(A+B K) \xi_{k}$ are mean-square stable [10], [11]. The uncertain system

$$
x_{k+1}=\Phi x_{k}+B c_{k}+w
$$

then generates predicted state trajectories, where $\Phi=A+B K$. Define $\Phi^{(j)}=A^{(j)}+B^{(j)} K$, and let $c_{k}=0$ for $k \geq N$. The sequence $c_{0}, \ldots, c_{N-1}$ is set by the controller, with the integer $N$ referred to as the prediction horizon.

At some prediction time $k$, the set of possible future states $x_{k}$ is a polyhedron with a number of vertices that grows exponentially with $k$. To avoid computing these sets we bound the predictions in a state tube, which is a sequence of polyhedra of fixed complexity. Introducing a parameter $\alpha_{k}$, we define the state tube cross sections as the sets:

$$
T_{k}=\left\{x: V x \leq \alpha_{k}\right\}
$$

The choice of $V$ is nontrivial and should be made by offline construction of an invariant set.

Assumption 3. For some $g$, the set $\{x: V x \leq g\}$ is robustly invariant for (II.1) under the control law $u(t)=K x(t)$.

We apply constraints so that the $T_{k}$ contain the predictions:

$$
\begin{aligned}
& T_{k} \subseteq\left\{x: \Phi^{(j)} x+B^{(j)} c_{k}+w^{(j)} \in T_{k+1}\right\}, \quad \forall j \\
& x_{0} \in T_{0} .
\end{aligned}
$$

In particular, since (III.4) implies $x_{k+1} \in T_{k+1}$ whenever $x_{k} \in$ $T_{k}$, for all $k$, we obtain $x_{k} \in T_{k}$ for $k \geq 0$ by induction. From (III.3), condition (III.5) is equivalent to $V x_{0} \leq \alpha_{0}$.

Constraints (II.4) and (II.5) can be applied to the predictions by requiring that the cross sections satisfy:

$$
\begin{aligned}
& T_{k} \subseteq\left\{x: F_{h} x+G_{h} u_{k} \leq \underline{1}\right\} \\
& T_{k} \subseteq\left\{x: \operatorname{Pr}\left[F_{p} x_{k+1} \leq \underline{1}\right] \geq p\right\}
\end{aligned}
$$

Using (III.1) and (III.2), and defining $\bar{F}_{h}=F_{h}+G_{h} K$, these conditions can be re-written as

$$
\begin{aligned}
& T_{k} \subseteq\left\{x: \bar{F}_{h} x+G_{h} c_{k} \leq \underline{1}\right\} \\
& T_{k} \subseteq\left\{x: \operatorname{Pr}\left[F_{p}\left(\Phi x+B c_{k}+w\right) \leq \underline{1}\right] \geq p\right\} .
\end{aligned}
$$

\section{LINEAR AND PROBABILISTIC CONSTRAINTS}

We extend a well-known condition for inclusion of polyhedra to the case where the containing set is defined by probabilistic constraints. The following lemma is from [12].

Lemma 1. Let $P_{i}=\left\{x: F_{i} x \leq b_{i}\right\}, i=1,2$, then $P_{1} \subseteq P_{2}$ if and only if there exists a matrix $H \geq 0$ satisfying:

$$
H F_{1}=F_{2}, \quad H b_{1} \leq b_{2} .
$$

The next result generalizes Lemma 1 to the case in which $P_{2}$ is defined by a linear chance constraint, which in general does not yield a polyhedral set.

Lemma 2. Let $P_{1}=\left\{x: F_{1} x \leq b_{1}\right\}$ and $P_{2}=\{x$ : $\left.\operatorname{Pr}\left[F_{2} x \leq b_{2}\right] \geq p\right\}$, with $F_{2}$ and $b_{2}$ defined as

$$
\left(F_{2}, b_{2}\right)=\sum_{i=1}^{\mu}\left(F_{2}^{\{i\}}, b_{2}^{\{i\}}\right) q_{i} .
$$

Then $P_{1} \subseteq P_{2}$ if and only if matrices $H^{\{i\}} \geq 0$ exist satisfying $H^{\{i\}} F_{1}=F_{2}^{\{i\}}$ for $i=1, \ldots, \mu$ and

$$
\operatorname{Pr}\left[\sum_{i=1}^{\mu}\left(H^{\{i\}} b_{1}-b_{2}^{\{i\}}\right) q_{i} \leq 0\right] \geq p .
$$

Proof: To prove sufficiency, suppose that $H^{\{i\}}, \ldots, H^{\{\mu\}}$ satisfy the conditions of the lemma. Then for any $x^{*}$ in $P_{1}$ we obtain $F_{2} x^{*}=\sum_{i=1}^{\mu} H^{\{i\}} q_{i} F_{1} x^{*} \leq \sum_{i=1}^{\mu} H^{\{i\}} q_{i} b_{1}$. Hence $\operatorname{Pr}\left[\sum_{i=1}^{\mu}\left(H^{\{i\}} b_{1}-b_{2}^{\{i\}}\right) q_{i} \leq 0\right] \geq p$ implies that $\operatorname{Pr}\left[F_{2} x^{*}-\right.$ $\left.b_{2} \leq 0\right] \geq p$ and therefore $x^{*} \in P_{2}$.

We prove necessity by construction. Assume $P_{1} \subseteq P_{2}$, then

$$
\operatorname{Pr}\left[\mu \leq b_{2}\right] \geq p, \quad \mu_{j}=\max _{x}\left\{\left(F_{2}\right)_{j} x: F_{1} x \leq b_{1}\right\},
$$

where $\left(F_{2}\right)_{j}$ is the $j$ th row of $F_{2}$ and $\mu_{j}$ is the $j$ th element of $\mu$. Strong duality holds for linear programs, so the dual of the linear program defining $\mu_{j}$ for any realization of $F_{2}$ implies

$$
\mu_{j}=\min _{h}\left\{h^{T} b_{1}: h^{T} F_{1}=\left(F_{2}\right)_{j}, h \geq 0\right\} .
$$

For any $i \in\{1, \ldots, \mu\}$, let $h_{j}^{\{i\}}$ denote the minimizing argument of the LP (IV.2) corresponding to $\left(F_{2}\right)_{j}=\left(F_{2}^{\{i\}}\right)_{j}$, and define $H^{\{i\}}$ as the matrix with $j$ th row equal to $\left(h_{j}^{\{i\}}\right)^{T}$. Then (IV.2) implies that $H^{\{i\}} \geq 0$ and $H^{\{i\}} F_{1}=$ $F_{2}^{\{i\}}$. Also from (IV.2), $\mu_{j}$ is equal to the $j$ th element of the vector $\sum_{i=1}^{\mu} H^{\{i\}} q_{i} b_{1}$, and therefore (IV.1) implies that $\operatorname{Pr}\left[\sum_{i=1}^{\mu}\left(H^{\{i\}} b_{1}-b_{2}^{\{i\}}\right) q_{i} \leq 0\right] \geq p$.

We now apply Lemmas 1 and 2 to the inclusions (III.4), (III.8) and (III.9). Analogously to $\Phi^{(j)}$, we define $\Phi^{\{i\}}=$ $A^{\{i\}}+B^{\{i\}} K$.

Proposition 3. $T_{k}=\left\{x: V x \leq \alpha_{k}\right\}$ satisfies (III.4) if there exist $H^{(j)} \geq 0, j=1, \ldots, \nu$, such that $H^{(j)} V=V \Phi^{(j)}$ and

$$
H^{(j)} \alpha_{k}+V B^{(j)} c_{k}+V w^{(j)} \leq \alpha_{k+1} .
$$


Proposition 4. $T_{k}=\left\{x: V x \leq \alpha_{k}\right\}$ satisfies (III.8) if there exists $H_{h} \geq 0$ such that $H_{h} V=\bar{F}_{h}$ and

$$
H_{h} \alpha_{k}+G_{h} c_{k} \leq \underline{1}
$$

Proposition 5. $T_{k}=\left\{x: V x \leq \alpha_{k}\right\}$ satisfies (III.9) if there exist $H_{p}^{\{i\}} \geq 0$ such that $H_{p}^{\{i\}} V=F_{p} \Phi^{\{i\}}, i=1, \ldots, \mu$, and with probability at least $p$,

$$
\sum_{i=1}^{\mu}\left(H_{p}^{\{i\}} \alpha_{k}+F_{p}\left(B^{\{i\}} c_{k}+w^{\{i\}}\right)\right) q_{i} \leq \underline{1} .
$$

To apply the set inclusions in the online MPC controller, a choice of ' $H$ ' matrix is made offline and the associated inequality constraint included in the online optimization, which is then a sufficient condition for the associated set inclusion. To relax the online constraints, these rows of these matrices can be chosen as the solutions of linear programs:

$$
\begin{aligned}
& \left(H^{(j)}\right)_{i}=\arg \min _{h}\left\{\underline{1}^{T} h: h^{T} V=(V)_{i} \Phi^{(j)}, h \geq 0\right\} \\
& \left(H_{h}\right)_{i}=\arg \min _{h}\left\{\underline{1}^{T} h: h^{T} V=\left(\bar{F}_{h}\right)_{i}, h \geq 0\right\} \\
& \left(H_{p}^{\{i\}}\right)_{l}=\arg \min _{h}\left\{\underline{1}^{T} h: h^{T} V=\left(F_{p}\right)_{l} \Phi^{\{i\}}, h \geq 0\right\} .
\end{aligned}
$$

When defined in this way, these matrices have several useful properties that simplify the online optimization.

Remark 2. If $\bar{F}_{h}$ appears as a block row of $V$ then $H_{h}=$ $\left[\begin{array}{llll}I & 0 & \cdots & 0\end{array}\right]$. This simplifies the constraints of Proposition 4 and occurs if $V$ defines the maximal robustly invariant set for $x_{k+1}=\Phi x_{k}+w$ subject to $\bar{F}_{h} x_{k} \leq \underline{1}$. Proposition 4 is then necessary and sufficient for constraint satisfaction. For details of how to compute this set, see [12] or [13].

Remark 3. Each of the matrices $H^{(j)}, H_{h}$ and $H_{p}^{\{i\}}$ defined by (IV.3), (IV.4) and (IV.5) is sparse with at most $\operatorname{dim}(x)$ nonzero entries in each row.

A terminal condition is required to ensure the online optimization remains feasible in closed-loop. In [3] a norm-bound was used, but here we use the less conservative condition

$$
H^{(j)} \alpha_{N}+V w^{(j)} \leq \alpha_{N} .
$$

This terminal constraint guarantees that states within the final tube cross section $T_{N}$ remain within $T_{N}$, effectively making it a terminal set for the MPC controller.

\section{QUADRATIC COST FUNCTION}

Due to additive uncertainty, it is not possible to use the cost (II.3) as the objective of the online MPC optimization directly because its optimal value may not be finite. Following the method of [9], we instead define the predicted cost as

$$
\mathbb{E}[J]=\sum_{k=0}^{\infty}\left(\mathbb{E}\left[L_{k}\right]-L_{s s}\right)
$$

where $L_{k}=x_{k}^{T} Q x_{k}+u_{k}^{T} R u_{k}$ for each $k=0,1, \ldots$ and $L_{s s}=\lim _{k \rightarrow \infty} \mathbb{E}\left[L_{k}\right]$. This limit is finite if $\xi_{k+1}=\Phi \xi_{k}$ is mean-square stable [9]; hence the predicted cost $\mathbb{E}[J]$ is finite.

Let $\underline{c}=\left[\begin{array}{llll}c_{0}^{T} & c_{1}^{T} & \cdots & c_{N-1}^{T}\end{array}\right]^{T}$ and $\zeta_{0}=\left[\begin{array}{ll}x_{0}^{T} & \underline{c}^{T}\end{array}\right]^{T}$ and define $\Psi=\left[\begin{array}{cc}\Phi & B E \\ 0 & T\end{array}\right], \quad \bar{Q}=\left[\begin{array}{cc}Q+K^{T} R K & K^{T} R E \\ E^{T} R K & E^{T} R E\end{array}\right], \quad \bar{w}=\left[\begin{array}{c}w \\ 0\end{array}\right]$ where $E$ and $T$ are the matrices such that $c_{0}=E \underline{c}$ and $T \underline{c}=$ $\left[\begin{array}{lllll}c_{1}^{T} & c_{2}^{T} & \cdots & c_{N-1}^{T} & 0\end{array}\right]^{T}$. Then the predicted sequences of states and control inputs are generated by $\zeta_{k+1}=\Psi \zeta_{k}+\bar{w}$.

Proposition 6. The cost (V.1) is given by

$$
\mathbb{E}[J]=\left[\begin{array}{c}
\zeta_{0} \\
1
\end{array}\right]^{T}\left[\begin{array}{cc}
P & v \\
v^{T} & \pi
\end{array}\right]\left[\begin{array}{c}
\zeta_{0} \\
1
\end{array}\right]
$$

where $P, v$ and $\pi$ solve the Lyapunov equation

$$
\left[\begin{array}{cc}
P & v \\
v^{T} & \pi
\end{array}\right]-\mathbb{E}\left(\left[\begin{array}{cc}
\Psi & \bar{w} \\
0 & 1
\end{array}\right]^{T}\left[\begin{array}{cc}
P & v \\
v^{T} & \pi
\end{array}\right]\left[\begin{array}{cc}
\Psi & \bar{w} \\
0 & 1
\end{array}\right]\right)=\left[\begin{array}{cc}
\bar{Q} & 0 \\
0 & -L_{s s}
\end{array}\right] .
$$

Given the linear dependence of $(\Psi, \bar{w})$ on $q$, it follows immediately that $P$ is the solution of the Lyapunov equation

$$
P-\sum_{i, j}\left(\Psi^{\{i\}}\right)^{T} P \Psi^{\{j\}} \mathbb{E}\left[q_{i} q_{j}\right]=\bar{Q}
$$

and $v$ is the solution of the linear equations

$$
\left(I-\sum_{i=1}^{\mu} \Psi^{\{i\}} \mathbb{E}\left[q_{i}\right]\right)^{T} v=\sum_{i, j}\left(\Psi^{\{i\}}\right)^{T} P \bar{w}^{\{j\}} \mathbb{E}\left[q_{i} q_{j}\right],
$$

where $\Psi^{\{i\}}$ and $\bar{w}^{\{i\}}$ are defined by replacing $\Phi, B$ and $w$ with $\Phi^{\{i\}}, B^{\{i\}}$ and $w^{\{i\}}$ in the definition of $\Psi$ and $\bar{w}$.

\section{Chance-COnstrained MPC OPTIMIZation}

The constraints of Section IV can be used to construct a recursively feasible MPC law. The control applied at each time is $u_{0}=K x_{0}+c_{0}$, where $c_{0}$ is given by the solution of an optimization problem. This online optimization consists of minimizing the predicted cost defined in Section V subject to contraints deriving from Propositions 3, 4 and 5, with the chance constraint applied only at time $k=0$ and a correspondng robust constraint applied for $k=1, \ldots, N$. Note that at time $k=0$ we may choose $\alpha_{0}=V x_{0}$.

Optimization 1. Chance-constrained tube MPC:

$$
\underset{\left\{c_{k}, \alpha_{k}\right\}}{\operatorname{minimize}} \zeta_{0}^{T} P \zeta_{0}+2 v^{T} \zeta_{0}
$$

subject to, for all $j=1,2, \ldots, \nu$ :

initial constraints :

$H^{(j)} V x_{0}+V B^{(j)} c_{0}+V w^{(j)} \leq \alpha_{1}, \quad H_{h} V x_{0}+G_{h} c_{0} \leq \underline{1}$, $\operatorname{Pr}\left[\sum_{i=1}^{\mu}\left(H_{p}^{\{i\}} V x_{0}+F_{p}\left(B^{\{i\}} c_{0}+w^{\{i\}}\right)\right) q_{i} \leq \underline{1}\right] \geq p$

tube constraints, for $k=1,2, \ldots, N-1$ :

$H^{(j)} \alpha_{k}+V B^{(j)} c_{k}+V w^{(j)} \leq \alpha_{k+1}, \quad H_{h} \alpha_{k}+G_{h} c_{k} \leq \underline{1}$,

$\sum_{i=1}^{\mu}\left(H_{p}^{\{i\}} \alpha_{k}+F_{p}\left(B^{\{i\}} c_{k}+w^{\{i\}}\right)\right) q_{i}^{(j)} \leq \underline{1}$

terminal constraints :

$H^{(j)} \alpha_{N}+V w^{(j)} \leq \alpha_{N}, \quad H_{h} \alpha_{N} \leq \underline{1}$,

$\sum_{i=1}^{\mu}\left(H_{p}^{\{i\}} \alpha_{N}+F_{p} w^{\{i\}}\right) q_{i}^{(j)} \leq \underline{1}$,

If the one-step ahead chance constraint at prediction time $k=0$ is replaced with its robust counterpart (as used for $k=1, \ldots, N-1$ ), then Optimization 1 reduces to a robust controller, similar to that of [4]. 


\section{SAMPLE-BASED MPC ALGORITHM}

This section considers implementing Optimization 1 using random samples, $q^{[1]}, \ldots, q^{[n]}$ drawn from the distribution of $q$ at each time $t$. The chance constraint may be approximated by specifying that it should hold for at least $n-r$ samples. For the rest of the prediction horizon, the constraint is applied robustly. To determine the $r$ samples allowed to violate constraints we use the following heuristic: if an approximate solution is available, then a good choice of samples to ignore are those that have the greatest constraint function values.

This motivates an algorithm where successively better approximations to the solution of Problem 1 are generated by solving with a fixed set of samples, sorting those samples, and solving again with the most restrictive samples removed. To facilitate this we introduce slack variables, denoted $s^{[l]}$, $l=1,2, \ldots, n$, leading to the quadratic program:

Optimization 2. Stochastic tube MPC with sampling:

$\underset{\left\{c_{k}, \alpha_{k}, s^{[l]}\right\}}{\operatorname{minimize}} \zeta_{0}^{T} P \zeta_{0}+2 v^{T} \zeta_{0}$

subject to, for $j=1,2, \ldots, \nu$ :

initial constraints :

$H^{(j)} V x_{0}+V B^{(j)} c_{0}+V w^{(j)} \leq \alpha_{1}, \quad H_{h} V x_{0}+G_{h} c_{0} \leq \underline{1}$, sampled constraints, for $l=1,2, \ldots, n$ :

$\sum_{i=1}^{\mu}\left(H_{p}^{\{i\}} V x_{0}+F_{p}\left(B^{\{i\}} c_{0}+w^{\{i\}}\right)\right) q_{i}^{[l]}+s^{[l]}=\underline{1}$,

tube constraints, for $k=1,2, \ldots, N-1$ :

$H^{(j)} \alpha_{k}+V B^{(j)} c_{k}+V w^{(j)} \leq \alpha_{k+1}, \quad H_{h} \alpha_{k}+G_{h} c_{k} \leq \underline{1}$,

$\sum_{i=1}^{\mu}\left(H_{p}^{\{i\}} \alpha_{k}+F_{p}\left(B^{\{i\}} c_{k}+w^{\{i\}}\right)\right) q_{i}^{(j)} \leq \underline{1}$,

terminal constraints :

$H^{(j)} \alpha_{N}+V w^{(j)} \leq \alpha_{N}, \quad H_{h} \alpha_{N} \leq \underline{1}$,

$\sum_{i=1}^{\mu}\left(H_{p}^{\{i\}} \alpha_{N}+F_{p} w^{\{i\}}\right) q_{i}^{(j)} \leq \underline{1}$,

slack variable conditions :

$s^{[l]} \geq 0, \quad \forall l \in \mathcal{I}, \quad|\mathcal{I}| \geq n-r$

The online algorithm involves solving Optimization 2 and refining the index set $\mathcal{I}$. This procedure is repeated to improve the accuracy of the solution. In contrast to the approach of [8], sampled constraints are employed only for the first prediction time $k=1$ rather than over the whole horizon. This may be conservative, but guarantees recursive feasibility.

Algorithm 1. Sample removal. At times $t=0,1, \ldots$ :

1) Draw samples $q^{[1]}, \ldots, q^{[n]}$ from the distribution of $q$.

2) Set $\mathcal{I}=\mathcal{I}_{l}=\{1,2, \ldots, n\}$.

3) Solve Optimization 2 using $\mathcal{I}$.

4) Set $m^{[l]}$ as the minimum element of $s^{[l]}$ for each $l$.

5) Set $\mathcal{I}$ to contain the $n-r$ indices ' $l$ ' with greatest $m^{[l]}$.

6) If $\mathcal{I}=\mathcal{I}_{l}$ stop. Otherwise set $\mathcal{I}_{l}=\mathcal{I}$ and return to 3 ).

Because of the nonconvexity of Optimization 2, the stopping criterion is necessarily heuristic, but in practice Algorithm 1 converges in a few iterations when the index set $\mathcal{I}$ is repeated.

\section{Closed-LOOP PROPERTIES}

Due to the bounding of predictions at time $k=N$ in the final tube cross-section $\mathcal{T}_{N}$, it is possible to give guarantees of feasibility and stability in closed-loop operation.

Theorem 7. Optimization 2 and Algorithm 1 define a recursively feasible MPC law. That is, if it is feasible at time $t$, then it remains feasible at all subsequent times in closed-loop.

Proof: Let $\left(c_{0}^{\prime}, \ldots, c_{N-1}^{\prime}\right),\left(\alpha_{0}^{\prime}, \ldots, \alpha_{N}^{\prime}\right)$ denote a feasible point at any given time $t$. We will show that $\left(c_{1}^{\prime}, \ldots, c_{N-1}^{\prime}, 0\right)$ and $\left(\alpha_{1}^{\prime}, \ldots, \alpha_{N}^{\prime}, \alpha_{N}^{\prime}\right)$ are feasible at time $t+1$ with $\mathcal{I}=$ $\{1,2, \ldots, n\}$. For the terminal constraints

$$
\begin{aligned}
& H^{(j)} \alpha_{N}+V w^{(j)} \leq \alpha_{N}, \quad H_{h} \alpha_{N} \leq 1, \\
& \sum_{i=1}^{\mu}\left(H_{p}^{\{i\}} \alpha_{N}+F_{p} w^{\{i\}}\right) q_{i}^{(j)} \leq \underline{1} .
\end{aligned}
$$

we observe that at time $t+1$ these are identical to the terminal constraints from time $t$, and therefore are satisfied. In a similar manner, the tube constraints

$$
\begin{aligned}
& H^{(j)} \alpha_{k}+V B^{(j)} c_{k}+V w^{(j)} \leq \alpha_{k+1}, \quad H_{h} \alpha_{k}+G_{h} c_{k} \leq 1, \\
& \sum_{i=1}^{\mu}\left(H_{p}^{\{i\}} \alpha_{k}+F_{p}\left(B^{\{i\}} c_{k}+w^{\{i\}}\right)\right) q_{i}^{(j)} \leq \underline{1}
\end{aligned}
$$

are trivially satisfied for $k=1, \ldots, N-2$ because they are identical to constraints used at time $t$. For $k=N-1$, they are identical to the terminal conditions used at time $t$, noting that our chosen ' $c$ ' is zero, and are also satisified.

The remaining constraints are the initial constraints, sampled constraints and slack variable conditions. If $\mathcal{I}=$ $\{1,2, \ldots, n\}$, then $|\mathcal{I}| \geq n-r$ is satisified and we may eliminate the slack variables to leave

$$
\begin{aligned}
& H^{(j)} V x_{0}+V B^{(j)} c_{0}+V w^{(j)} \leq \alpha_{1}, \quad H_{h} V x_{0}+G_{h} c_{0} \leq \underline{1}, \\
& \sum_{i=1}^{\mu}\left(H_{p}^{\{i\}} V x_{0}+F_{p}\left(B^{\{i\}} c_{0}+w^{\{i\}}\right)\right) q_{i}^{[l]} \leq \underline{1},
\end{aligned}
$$

where the sampled constraint applies for all $l=1, \ldots, n$. Because the initial state $x_{0}$ at time $t+1$ necessarily lies within the tube cross-section $T_{1}$ from time $t$, we have $V x_{0} \leq \alpha_{1}^{\prime}$. Noting that all elements of the ' $H$ ' matrices are nonnegative, comparing the first two constraints with the $k=1$ tube constraints from time $t$ leads immediately to the conclusion that they are satisfied, as we have $H V x_{0} \leq H \alpha_{1}^{\prime}$ where $H$ is either $H^{(j)}$ or $H_{h}$. Finally, the sampled constaint is satisfied for all $l$ because we have $H_{p}^{\{i\}} V x_{0} \leq H_{p}^{\{i\}} \alpha_{1}^{\prime}$ for all $i$ and all samples $q^{[l]} \in \operatorname{Co}\left\{q^{(1)}, \ldots, q^{(\nu)}\right\}$ by Assumption 2 .

Theorem 8. The system (II.1) under the control law $u(t)=$ $K x(t)+c_{0}$, where $c_{0}$ is the first element of $\underline{c}$ defined by Optimization 2 and Algorithm 1, is stable in the sense that

$$
\lim _{\tau \rightarrow \infty} \frac{1}{\tau} \sum_{t=0}^{\tau-1} \mathbb{E}_{0}[L(t)] \leq L_{s s}
$$

where $L(t)=x(t)^{T} Q x(t)+u(t)^{T} R u(t)$, and $x(t), u(t)$ are the state and input of the closed loop system at time $t$.

Proof: Let $\mathcal{V}(x(t))$ denote the predicted cost (V.1) evaluated at the solution of Optimization 2 at time $t$. Then the 
feasibility and the suboptimality of the sequences $T \underline{c}$ and $\left(\alpha_{1}, \ldots, \alpha_{N}, \alpha_{N}\right)$ at time $t+1$ implies

$$
\mathbb{E}_{t}[\mathcal{V}(x(t+1))] \leq \mathcal{V}(x(t))-\left(\mathbb{E}_{t}[L(t)]-L_{s s}\right)
$$

Taking expectations and summing each side of this inequality over $t=0,1 \ldots, \tau-1$, we then obtain

$$
\frac{1}{\tau} \sum_{t=0}^{\tau-1} \mathbb{E}_{0}[L(t)] \leq L_{s s}+\frac{1}{\tau}\left(\mathcal{V}(x(0))-\mathbb{E}_{0}[\mathcal{V}(x(\tau))]\right) .
$$

However by Proposition 6 and (V.3), $\mathcal{V}(x)$ is necessarily bounded from below for all feasible $x$, so that $\lim _{\tau \rightarrow \infty} \frac{1}{\tau} \mathbb{E}_{0}[\mathcal{V}(x(\tau))]=0$, and the bound given by (VIII.1) therefore follows when passing to the limit $\tau \rightarrow \infty$.

We now appeal to the 'sampling and discarding' theory of random convex programs found in [14], [15] to show that the chance constraint (II.5) holds with high confidence in closedloop, in the sense that we are unlikely to draw samples $q^{[l]}$ such that (II.5) does not hold. To state the result, let $\mathcal{F}_{n, s}(p)$ denote the binomial distribution of $s$ or fewer successes of probability $p$ in $n$ trials. We also use the notion of a support constraint, which is a sampled constraint for which the optimal cost decreases if it is removed [15, Definition 2.1].

Theorem 9. The probability that the chance constraint (II.5) is violated at any time $t$ in closed-loop is at most

$$
\varepsilon=(\underset{r}{r+\operatorname{dim}(u)-1}) \mathcal{F}_{n, r+\operatorname{dim}(u)-1}(1-p) .
$$

Proof: Noting that satisfaction of the chance constraint in Optimization 1 implies (II.5) by Proposition 5, and that Optimization 2 is the sampled counterpart of Optimization 1 , the result will follow from [15, Theorem 4.1] if the number of support constraints of Optimization 2 is bounded by $m=\operatorname{dim}(u)$ (in [15] the best such bound is used, which is termed Helly's dimension).

Suppose that Optimization 2 has $m+d$ support constraints. Considering a rearrangement of the sampled constraints

$$
\left(\sum_{i=1}^{\mu} q_{i}^{[l]} F_{p} B^{\{i\}}\right) c_{0} \leq \underline{1}-\sum_{i=1}^{\mu} q_{i}^{[l]}\left(H_{p}^{\{i\}} V x_{0}+F_{p} w^{\{i\}}\right)
$$

and omitting all rows for each value of $l$ which do not yield support constraints, we observe that the support constraints may be written as $S c_{0} \leq v$, in which $S$ is a $(m+d) \times m$ matrix and $v \in \mathbb{R}^{m+d}$. Due to convexity, these are satisfied with equality at the solution $c_{0}^{*}$ and hence $S c_{0}^{*}=v$. As a solution $c_{0}^{*}$ exists, these $m+d$ linear equations in $m$ variables must have at least $d$ redundancies and removal of the corresponding support constraints from Optimization 2 will not affect its solution. But this contradicts the supposition that there are $m+d$ support constraints, so Optimization 2 has at most $m=\operatorname{dim}(u)$ support constraints and the result follows.

\section{NUMERICAL EXAMPLE}

Consider the system of (II.1)

$$
x(t+1)=A(t) x(t)+B(t) u(t)+w(t)
$$

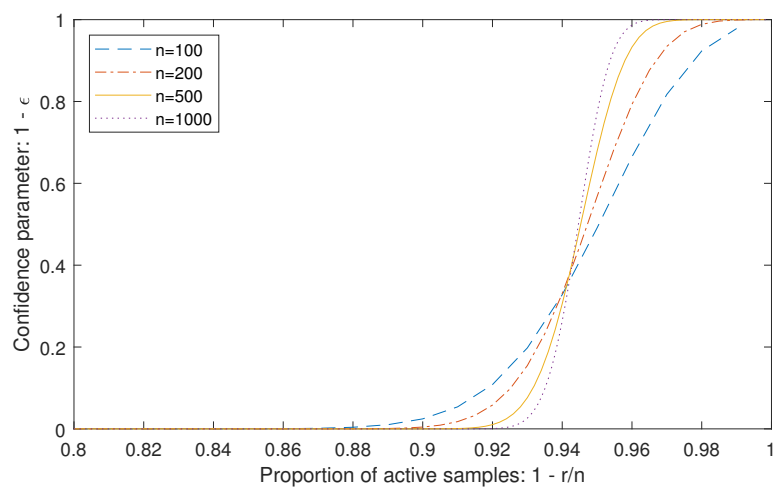

Fig. 1: Confidence value for example, $\operatorname{dim}(u)=1$

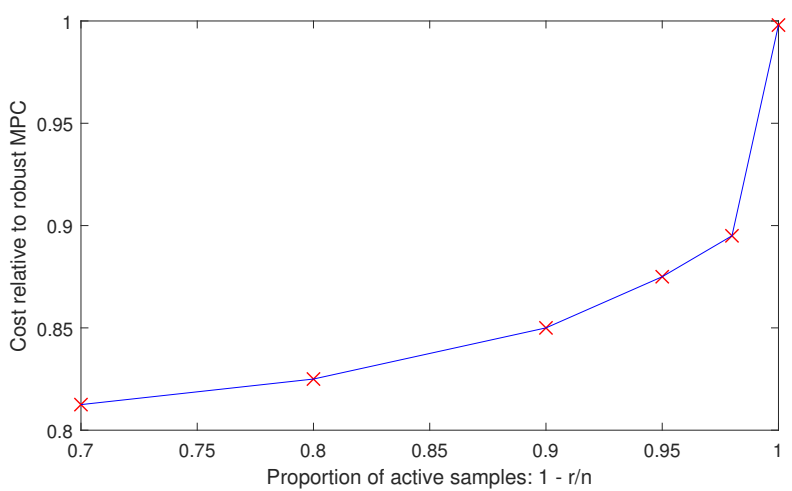

Fig. 2: Cost improvement for example, $n=250$

with nominal (expected-value) system matrices:

$$
A_{0}=\left[\begin{array}{cc}
-1.9 & -1.4 \\
0.7 & 0.5
\end{array}\right], \quad B_{0}=\left[\begin{array}{c}
1 \\
-0.25
\end{array}\right] \text {. }
$$

We define random variables

$$
\begin{aligned}
& A=A_{0}+\Delta_{A}^{\{1\}} q_{1}+\Delta_{A}^{\{2\}} q_{2}+\Delta_{A}^{\{3\}} q_{3} \\
& B=B_{0}+\Delta_{B}^{\{1\}} q_{4}+\Delta_{B}^{\{2\}} q_{5}, w=w^{\{1\}} q_{6}+w^{\{2\}} q_{7}
\end{aligned}
$$

where $q_{1}, \ldots, q_{7}$ are independent random scalars uniformly distributed on the interval $[0,1]$, and

$$
\begin{gathered}
\Delta_{A}^{(1)}=\left[\begin{array}{rr}
0.01 & 0.05 \\
-0.05 & -0.01
\end{array}\right], \quad \Delta_{A}^{(2)}=\left[\begin{array}{cc}
-0.01 & -0.05 \\
0 & -0.01
\end{array}\right] \\
\Delta_{A}^{(3)}=\left[\begin{array}{cc}
0 & 0 \\
0.05 & 0.02
\end{array}\right], \quad \Delta_{B}^{(1)}=\left[\begin{array}{r}
0.03 \\
-0.02
\end{array}\right] \\
\Delta_{B}^{(2)}=\left[\begin{array}{r}
-0.03 \\
0.02
\end{array}\right], \quad w_{1}=\left[\begin{array}{r}
0.2 \\
-0.2
\end{array}\right], \quad w_{2}=\left[\begin{array}{r}
-0.2 \\
0.2
\end{array}\right] .
\end{gathered}
$$

The support of $\left(q_{1}, \ldots, q_{7}\right)$ is therefore the unit hypercube with $\nu=128$ vertices. The cost matrices and probabilistic constraint are defined as:

$Q=\left[\begin{array}{ll}1 & 0 \\ 0 & 1\end{array}\right], \quad R=1, \quad \operatorname{Pr}_{t}[[-0.51] x(t+1) \leq 1] \geq 0.9$.

The stochastic MPC algorithm of this paper, and its robust analog from [4], were used with initial condition $x_{0}=\left[\begin{array}{ll}4 & 4\end{array}\right]^{T}$, prediction horizon $N=4$ and taking $n=250$ samples of $q$. The feedback gain $K$ was chosen to be the LQ-optimal for 


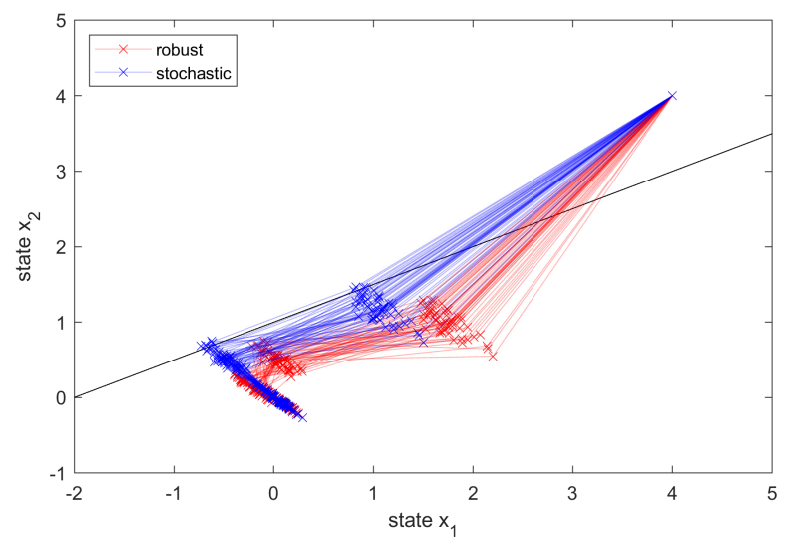

Fig. 3: Closed-loop trajectories for robust MPC [4] and stochastic MPC (Alg. 1) for 50 realizations of $\{q(0), q(1), \ldots\}$

\begin{tabular}{l|ccc} 
MPC Algorithm & Robust [4] & $\begin{array}{c}\text { Algorithm 1 } \\
(r=14)\end{array}$ & $\begin{array}{c}\text { Scenario [8] } \\
(r=14)\end{array}$ \\
\hline Mean closed-loop cost & 244.19 & 208.85 & 208.55 \\
\% satisfied constraints $t=1$ & 100.0 & 95.4 & 93.2 \\
\% satisfied constraints $t=2$ & 100.0 & 96.4 & 100.0 \\
Mean computation time /ms & 79.1 & 167.4 & 210.0
\end{tabular}

TABLE I: Robust and stochastic MPC with sample removal

the pair $\left(A_{0}, B_{0}\right): K=\left[\begin{array}{ll}1.31 & 0.97\end{array}\right]$, and $V$ was constructed by finding the maximal robustly invariant set respecting the constraint $[-0.51] x \leq 1$, which is defined by 22 linear inequalities. For this example, $\operatorname{dim}(u)=1$ and Theorem 9 can be applied to give the relationship between $n, r$ and $\epsilon$. This is shown in Figure 1 for $p=0.9$. In this case, a confidence value of $99 \%$ mandated that $r=14$ samples could violate the sampled constraint. Simulations were carried out in MATLAB, using Yalmip [16] and the QP solver Gurobi. The resulting state-space trajectories are shown in Figure 3.

Table I compares the closed loop properties of the robust and stochastic implementations for 500 realizations of model uncertainty, as well as comparing with the 'scenario' approach of [8] when using the same heuristic for sample removal. The mean closed loop cost is $15 \%$ lower in the stochastic case than the robust. The proportion of trajectories satisfying constraints (95.4\%) implies conservativism in the stochastic MPC with respect to the probabilistic constraint, which requires $[-0.51] x(t+1) \leq 1$ to be satisfied for $90 \%$ of uncertainty realisations. However, this is expected from the confidence value of $99 \%$, which requires $1-r / n=0.944$ for $n=250$. As more samples are used, $1-r / n$ approaches 0.9 asymptotically. For a given $n$, Algorithm 1 appears slightly more conservative than the scenario MPC of [8] in terms of mean closed-loop cost, but unlike scenario MPC, Algorithm 1 guarantees feasibility in closed-loop.

\begin{tabular}{l|ccc} 
MPC Algorithm & Robust [4] & $\begin{array}{c}\text { Algorithm 1 } \\
(r=0)\end{array}$ & $\begin{array}{c}\text { Scenario [8] } \\
(r=0)\end{array}$ \\
\hline Mean closed-loop cost & 244.19 & 214.09 & 213.35 \\
\% satisfied constraints $t=1$ & 100.0 & 97.6 & 96.8 \\
\% satisfied constraints $t=2$ & 100.0 & 97.8 & 100.0 \\
Mean computation time /ms & 79.1 & 74.6 & 29.6
\end{tabular}

TABLE II: Robust and stochastic MPC without sample removal
It is also of interest to compare with the case that sampling is used, but with no sample removal $(r=0)$. Applying the chance constraint with $p=0.9$ and $99 \%$ confidence requires $n=44$ samples. Table II shows that Algorithm 1 gives a $12 \%$ improvement in closed-loop cost over Robust MPC but requires similar computation time. It is notable that the scenario MPC of [8] has a lower computation time than Algorithm 1 when $n=44$, but a higher computation time when $n=250$. The reason is that the number of constraints in the optimization is $O(n N)$ for the scenario MPC of [8], but is $O(n+\nu(N-1))$ with $\nu>44$ in Optimization 2 as sampled constraints are only applied at the first prediction time.

Using robust MPC in this example leads to conservativism and a significant increase in closed-loop cost compared to the stochastic MPC algorithms. This is a result of having several independent sources of uncertainty: although each is uniformly distributed, the one-step ahead probability density function resulting from them will quickly fall to a small value as distance from the mode increases. Figure 2 shows the 'normalized cost' given by division of the mean stochastic MPC cost by the mean robust MPC cost against the proportion of active samples. The improvement is significant even when $1-r / n=0.98$.

\section{REFERENCES}

[1] Y. Lee and B. Kouvaritakis, "Robust receding horizon predictive control for systems with uncertain dynamics and input saturation," Automatica, vol. 36, no. 10, pp. 1497-1504, 2000.

[2] D. Mayne and J. Rawlings, "Model predictive control: Theory and design," Nob Hill Publishing, 2009.

[3] J. Fleming, M. Cannon, and B. Kouvaritakis, "Stochastic tube MPC for LPV systems with probabilistic set inclusion conditions," in 53rd IEEE Conf. Decision and Control, 2014.

[4] J. Fleming, B. Kouvaritakis, and M. Cannon, "Robust tube MPC for linear systems with multiplicative uncertainty," IEEE Transactions on Automatic Control, vol. 60, no. 4, pp. 1087-1092, 2015.

[5] B. Kouvaritakis, M. Cannon, S. Raković, and Q. Cheng, "Explicit use of probabilistic distributions in linear predictive control," Automatica, vol. 46, no. 10, pp. 1719-1724, 2010.

[6] M. Korda, R. Gondhalekar, J. Cigler, and F. Oldewurtel, "Strongly feasible stochastic model predictive control," in 50th IEEE Conf. Decision and Control, 2011, pp. 1245-1251.

[7] G. Calafiore and L. Fagiano, "Stochastic model predictive control of LPV systems via scenario optimization," Automatica, vol. 49, no. 6, pp. 1861-1866, 2013.

[8] G. Schildbach, L. Fagiano, C. Frei, and M. Morari, "The scenario approach for stochastic model predictive control with bounds on closedloop constraint violations," Automatica, vol. 50, no. 12, pp. 3009-3018, 2014.

[9] M. Cannon, B. Kouvaritakis, and X. Wu, "Probabilistic constrained MPC for multiplicative and additive stochastic uncertainty," IEEE Transactions on Automatic Control, vol. 54, no. 7, pp. 1626-1632, 2009.

[10] H. Kushner, Stochastic stability and control. Academic Press, 1967.

[11] S. Boyd, L. El Ghaoui, E. Feron, and V. Balakrishnan, Linear Matrix Inequalities in System and Control Theory. SIAM, 1994.

[12] F. Blanchini and S. Miani, Set-theoretic methods in control. Springer, 2007.

[13] B. Pluymers, J. Rossiter, J. Suykens, and B. De Moor, "The efficient computation of polyhedral invariant sets for linear systems with polytopic uncertainty," in American Control Conference, 2005, pp. 804-809.

[14] M. Campi and S. Garatti, "A sampling-and-discarding approach to chance-constrained optimization: feasibility and optimality," Journal of Optimization Theory and Applications, vol. 148, pp. 257-280, 2011.

[15] G. C. Calafiore, "Random convex programs," SIAM Journal on Optimization, vol. 20, no. 6, pp. 3427-3464, 2010.

[16] J. Löfberg, "YALMIP : A toolbox for modeling and optimization in MATLAB," in In Proceedings of the CACSD Conference, Taipei, Taiwan, 2004. 